\title{
Expectation values of four-quark operators in pions
}

\author{
E.G. Drukarev, M.G. Ryskin, V.A. Sadovnikova, \\ Petersburg Nuclear Physics Institute \\ Gatchina, 188300 St. Petersburg, Russia \\ and \\ Amand Faessler \\ Institute of Theoretical Physics, University of Tuebingen, Morgenstelle 14, D-72076, Tuebingen, Germany \\ (today)
}

\begin{abstract}
The values of four-quark operators averaged over pions are expressed through those averaged over vacuum. The specific values are obtained in the framework of the factorization assumption. For the condensates of the light quarks of the same flavour $\bar{q} \Gamma q \bar{q} \Gamma q$ the scalar condensate is shown to be an order of magnitude larger than the other ones. The condensates containing the strange quarks $\bar{q} q \bar{s} s$ appear to be only about twice smaller than those of the light quarks. The degeneracy of the ground state in the Nambu-Jona-Lasinio model is shown explicitly.
\end{abstract}

\section{INTRODUCTION}

The matrix elements of four-quark operators in hadronic states provide information on the structure of the quarkantiquark sea in the hadrons describing the correlations of $\bar{q} q$ pairs. Under certain reasonable assumptions the expectation value of the scalar quark operator $\bar{q} q$ was found in [1]- [3] to be equal (up to normalization factors) to the total number of quarks and antiquarks $n_{h}$ in the hadron. The known expectation values of $\bar{q} q$ in nucleons and pions [4] correspond to $n_{N} \approx 8$ and $n_{\pi} \approx 12$, exceeding the number of the valence quarks. This signals the large numbers of the sea quarks even at low energies.

Another application is the QCD sum rules in nuclear matter. In-medium values of the four-quark condensates is an important ingredient of the approach [5, 6 . In the gas approximation it is expressed through the expectation value in the nucleons.

As it stands now, the only calculation of the four-quark condensate in hadrons is that carried out by Celenza et al. [7]. In [7] the condensate in the nucleons was obtained in the framework of the Nambu and Jona-Lasinio model. Some data on its value in nucleons were obtained by Johnson and Kisslinger [8] by considering the QCD sum rules for nucleons and isobars.

Here we present calculation of the expectation values of the four-quark operators in pions. Besides providing information on the structure of pions, the analysis is the intermediate step in the calculation of the expectation values in nucleons in the framework of models where the quark-antiquark sea is contained in the pion cloud. Some of the models analyzed in [9] as well as perturbative chiral quark model [10], provide the examples.

The general form of the four-quark expectation value can be presented as

$$
Q_{12}=\left\langle\pi\left|\bar{q} T_{1} q \cdot \bar{q} T_{2} q\right| \pi\right\rangle
$$

with the subscripts of the matrices $T_{1,2}$ including spin, colour and flavour. By using the reduction formula obtained by Lehmann, Symanzik and Zimmerman (LSZ) [11,12] we present the matrix elements of Eq.(1) through the expectation values in vacuum

$$
Q_{12}=\frac{1}{f_{\pi}^{2}} \sum_{i, j}\left\langle 0\left|\bar{q} T_{i} q \cdot \bar{q} T_{j} q\right| 0\right\rangle
$$

with $f_{\pi} \approx 93 \mathrm{MeV}$ being the pion decay constant. Further calculations are carried out in the framework of a factorization hypothesis for the vacuum expectation values. This hypothesis was first formulated by Shifman et al. [13] and was advocated recently in [14. In this approximation the expectation values of the light quark operators are expressed through the well known value [15] of the condensate

$$
\langle 0|\bar{q} q| 0\rangle=-\frac{m_{\pi}^{2} f_{\pi}^{2}}{m_{u}+m_{d}}
$$

with $m_{\pi, u, d}$ standing for the masses of the pion and of the light quarks. For example, if " $q$ " is " $u$ " or " $d$ " quark

$$
\left\langle\pi\left|(\bar{q} q)^{2}\right| \pi\right\rangle \approx \frac{-2(\langle 0|\bar{q} q| 0\rangle)^{2}}{f_{\pi}^{2}}
$$


for any state of the pion isotope triplet.

The negative value of the condensate corresponds to a domination of "disconnected terms" when one of two $\bar{q} q$ pairs comes from the vacuum. The $\bar{q} q$ pairs in the pion are found to be correlated strongly, with the probability of the coexistence of two pairs being much smaller than in the independent pairs picture.

The four-quarks interactions are presented in Nambu and Jona-Lasinio model [16], emerging in its simplest SU(2) version. The instanton induced t'Hooft interaction [17] is often included in the applications nowadays-see e.g. [18]. Being written in terms of the current quarks, these interactions $V_{1,2}$ correspond to the picture "before" the symmetry was spontaneously broken. Thus the result of averaging over the pion states $\left\langle\pi\left|V_{1}\right| \pi\right\rangle=\left\langle\pi\left|V_{2}\right| \pi\right\rangle=0$ is a reasonable one. This means that introducing the interactions $V_{1,2}$ does not change the energy of the ground state. Hence, the latter remains degenerate until the spontaneous symmetry breaking occurs.

\section{GENERAL EQUATIONS}

We define the expectation value of any operator $A$ in a hadron $h$ as

$$
\langle h|\widehat{A}| h\rangle=\left\langle h\left|\int d^{3} x[\widehat{A}(x)-\langle 0|\widehat{A}(x)| 0\rangle]\right| h\right\rangle
$$

with the vector of state $\langle h|$ normalized as $\langle h(k) \mid h(p)\rangle=2 p_{0} \delta(\mathbf{k}-\mathbf{p})$. Of course, the expectation value $\langle 0|\widehat{A}(x)| 0\rangle$ does not depend on $x$. Thus the matrix element $\langle h|\widehat{A}| h\rangle$ is defined as the excess of the density of the operator $A$ over the vacuum value, integrated over the volume of the hadron.

Due to the partial conservation of axial current (PCAC) the pion state vector can be expressed through that of the vacuum (see, e.g., [12])

$$
\left|\pi^{\alpha}(x)\right\rangle=\frac{1}{\sqrt{2} f_{\pi} m_{\pi}^{2}} \partial_{\mu} J_{\mu 5}^{\alpha}(x)|0\rangle
$$

with $\alpha$ being the isospin index while $J_{\mu s}^{\alpha}$ is the axial current of the light quarks with the corresponding quantum numbers:

$$
\begin{aligned}
& J_{\mu 5}^{-}(x)=\sum_{c} \bar{d}^{c}(x) \gamma_{\mu} \gamma_{5} u^{c}(x) ; \quad J_{\mu 5}^{+}(x)=\sum_{c} \bar{u}^{c}(x) \gamma_{\mu} \gamma_{5} d^{c}(x) ; \\
& J_{\mu 5}^{0}(x)=\sum_{c} \frac{\bar{u}^{c}(x) \gamma_{\mu} \gamma_{5} u^{c}(x)-\bar{d}^{c}(x) \gamma_{\mu} \gamma_{5} d^{c}(x)}{\sqrt{2}}
\end{aligned}
$$

with " $c$ " being the colour index.

By applying Eq.(5) for both $\langle\pi|$ and $|\pi\rangle$ states in the matrix element $\langle\pi|A| \pi\rangle$ of any operator $A$ one finds the reduction LSZ formula [11, 12]

$$
\left\langle\pi^{\alpha}|\widehat{A}| \pi^{\alpha}\right\rangle=\frac{1}{f_{\pi}^{2}}\left\langle 0\left|B^{\alpha}\right| 0\right\rangle
$$

with

$$
B^{\alpha}=\frac{1}{2 V} \int d^{3} x d y_{0} d z_{0} \delta\left(x_{0}-y_{0}\right) \delta\left(z_{0}-x_{0}\right)\left[\bar{Q}_{5}^{\alpha}\left(z_{0}\right),\left[Q_{5}^{\alpha}\left(y_{0}\right), A(x)\right]\right] .
$$

Here $V$ is the normalization volume, the commutator $[X, Y]=X Y-Y X$, while

$$
Q_{5}^{\alpha}\left(y_{0}\right)=\int d^{3} y J_{05}^{\alpha}(y)
$$

is the axial charge, corresponding to the current $J_{\mu 5}^{\alpha}$.

Note that application of Eq.(9) to the quark scalar operator $A=\bar{u} u+\bar{d} d$ yields [4]

$$
\langle\pi|\bar{u} u+\bar{d} d| \pi\rangle=\frac{2 m_{\pi}^{2}}{m_{u}+m_{d}}
$$

with the proper behavior in the chiral limit. It was shown in [19] that the dimensionless value, identified with the total number of quarks and antiquarks

$$
n_{\pi}=\frac{\langle\pi|\bar{u} u+\bar{d} d| \pi\rangle}{2 m_{\pi}}
$$

can be obtained by using the pion vector of state $|\tilde{\pi}\rangle$ with normalization $\langle\tilde{\pi}(k) \mid \tilde{\pi}(p)\rangle=\delta(\mathbf{k}-\mathbf{p})$. 


\section{THE FOUR-QUARK OPERATORS}

The general form of the operator, containing the four quark fields $q$ of a specific flavour is $A=q_{\alpha}^{a} \bar{q}_{\beta}^{b} q_{\gamma}^{c} \bar{q}_{\delta}^{d}$ with $\alpha, \beta, \gamma, \delta$ and $a, b, c, d$ being Lorentz and colour indices. In the applications the product of the fields is time-ordered. Thus, considering the product of the field operators at the same space-time point we treat it as the limit of a timeordered product.

Each of the products of the two-quark fields can be presented as

$$
q_{\alpha}^{a} \bar{q}_{\beta}^{b}=-\frac{1}{12} \sum_{X} \bar{q} \Gamma^{X} q \Gamma_{\alpha \beta}^{X} \delta_{a b}-\frac{1}{64} \sum_{X, \rho} \bar{q} \Gamma^{X} \lambda^{\rho} q \Gamma_{\alpha \beta}^{X} \lambda_{a b}^{\rho}
$$

with $\lambda^{\rho}(\rho=1 \ldots 8)$ standing for the standard Gell-Mann matrices normalized by the relation $\operatorname{Sp} \lambda^{\rho} \lambda^{\tau}=2 \delta^{\rho \tau}$. The 16 basic Dirac $4 \times 4$ matrices are

$$
\Gamma^{1}=I, \quad \Gamma_{\mu}^{2}=\gamma_{\mu}, \quad \Gamma^{3}=\gamma_{5}, \Gamma_{\mu}^{4}=\gamma_{\mu} \gamma_{5}, \quad \Gamma_{k}^{5}=\frac{1}{2}\left(\gamma_{\mu} \gamma_{\nu}-\gamma_{\nu} \gamma_{\mu}\right)
$$

with $\mu=1 \ldots 4, k=1 \ldots 6$.

Thus it is sufficient to study the condensates of the form

$$
S_{X Y}=\left\langle h\left|\sum_{a, b} \bar{q}^{a} \Gamma^{X} q^{a} \cdot \bar{q}^{b} \Gamma^{Y} q^{b}\right| h\right\rangle
$$

and

$$
R_{X Y}=\left\langle h\left|\sum_{a, a^{\prime}, b, b^{\prime}, \rho} \bar{q}^{a} \Gamma^{X} \lambda_{a a^{\prime}}^{\rho} q^{a^{\prime}} \cdot \bar{q}^{b} \Gamma^{Y} \lambda_{b b^{\prime}}^{\rho} q^{b^{\prime}}\right| h\right\rangle
$$

with the matrices $\Gamma(\lambda)$ acting on Lorentz (colour) indices.

For the expectation values of the operators, which are antisymmetric in the colour variables

$$
U_{X Y}=\left\langle h\left|\sum_{a, a^{\prime}, b, b^{\prime}} \bar{q}^{a} \Gamma^{X} q^{a^{\prime}} \cdot \bar{q}^{b} \Gamma^{Y} q^{b^{\prime}}\right| h\right\rangle\left(\delta_{a a^{\prime}} \cdot \delta_{b b^{\prime}}-\delta_{a b^{\prime}} \cdot \delta_{b a^{\prime}}\right)
$$

we find by using the properties of Gell-Mann matrices

$$
U_{X Y}=\frac{2}{3} S_{X Y}-\frac{1}{2} R_{X Y}
$$

with $S_{X Y}$ and $R_{X Y}$ being defined by Eqs. (15), (16).

The condensates determined by Eqs. (15), (16) contain the quarks of the same flavour. For the case of different

flavours of quarks: $u, d(u, d, s)$ one can present similar covariant expressions replacing $\Gamma^{X, Y}$ by $T_{i}^{X, Y}=\Gamma^{X, Y} \cdot t^{i}$ with $t^{i}$ acting on the isospin indices.

\section{EXPECTATION VALUES OF THE LIGHT QUARK OPERATORS}

Here we calculate the expectation values of the 4-quark operators which include " $u$ " and "d" quarks only. The commutators which enter Eq.(8) can be calculated by using the relation [16]

$$
\left\{q_{\alpha}^{a}(x), \bar{q}_{\beta}^{b}(y)\right\}=\left(\gamma_{0}\right)_{\alpha \beta} \delta_{a b} \delta(\mathbf{x}-\mathbf{y})
$$

(with $\{X, Y\}=X Y+Y X)$ at $x_{0}=y_{0}$, while the anticommutators between all the other quark operators turn to zero. Evaluation of the right-hand side (rhs) of Eq.(9) thus leads to the form

$$
\left\langle 0\left|B^{\alpha}\right| 0\right\rangle=\frac{1}{V}\left\langle 0\left|\int d^{3} x F^{\alpha}(x)\right| 0\right\rangle
$$

with $F^{\alpha}(x)$ being the product of four quark operators. Since the vacuum is uniform, the density $F^{\alpha}$ does not depend on $x$. Hence, we can put 


$$
B^{\alpha}=F^{\alpha}(0) .
$$

To illustrate how it works we present the calculation of the operator $B$, corresponding to the expectation value $\left\langle\pi^{-}|\bar{u} \Gamma u \cdot \bar{u} \Gamma u| \pi^{-}\right\rangle$with $\Gamma=\Gamma^{X}=\Gamma^{Y}$. The internal commutator in the rhs of Eq.(9) is

$$
\kappa=\int d^{3} y \sum_{a}\left[\bar{d}^{a}(y) \gamma_{0} \gamma_{5} u^{a}(y), \bar{u}(x) \Gamma u(x) \bar{u}(x) \Gamma u(x)\right] .
$$

Presenting explicitly $\bar{d}^{a} \gamma_{0} \gamma_{5} u^{a}=\sum_{\gamma, \alpha} \bar{d}_{\gamma}^{a}\left(\gamma_{0} \gamma_{5}\right)_{\gamma_{\alpha}} u_{\alpha}^{a}$ and $\bar{u} \Gamma u=\sum_{\beta, \delta, b} \bar{u}_{\beta}^{b} \Gamma_{\beta \delta} u_{\delta}^{b}$ we employ the fact that the anticommutator of the fields $u_{\alpha}^{a}$ and $\bar{u}_{\beta}^{b}$ is given by Eq.(19) while all the others ones are zero. Using also the relation $\left\{\gamma_{0}, \gamma_{5}\right\}=0$ we obtain

$$
\kappa=-\left\{\bar{d}(x) \gamma_{5} \Gamma u(x), \bar{u}(x) \Gamma u(x)\right\} .
$$

After similar calculation of the commutator $\left[\bar{Q}_{5}^{-}, \kappa\right]$ we find

$$
B^{-}\left((\bar{u} \Gamma u)^{2}\right)=-\bar{u} \Gamma u\left(\bar{u} \Gamma u+\bar{d} \gamma_{5} \Gamma \gamma_{5} d\right)-\bar{d} \gamma_{5} \Gamma u \bar{u} \Gamma \gamma_{5} d .
$$

Here we introduced notation $B^{\alpha}(A)$ for the operator $B$ related to operator $A$ by Eq.(9).

One can obtain in the same way

$$
B^{+}\left((\bar{u} \Gamma u)^{2}\right)=B^{-}\left((\bar{u} \Gamma u)^{2}\right)
$$

and

$$
B^{0}\left((\bar{u} \Gamma u)^{2}\right)=-\bar{u} \Gamma u\left(\bar{u} \Gamma u+\bar{u} \gamma_{5} \Gamma \gamma_{5} u\right)-\frac{1}{2} \bar{u}\left\{\gamma_{5}, \Gamma\right\} u \cdot \bar{u}\left\{\gamma_{5}, \Gamma\right\} u .
$$

For the mixed operator $A=\bar{u} \Gamma u \bar{d} \Gamma d$ we find

$$
B^{ \pm}(\bar{u} \Gamma u \bar{d} \Gamma d)=-\bar{u} \Gamma u \bar{d} \Gamma d-\frac{1}{2}\left(\bar{u} \Gamma u \bar{u} \gamma_{5} \Gamma \gamma_{5} u+\bar{u} \gamma_{5} \Gamma d \bar{d} \gamma_{5} \Gamma u+(u \leftrightarrow d)\right),
$$

while

$$
B^{0}(\bar{u} \Gamma u \bar{d} \Gamma d)=-\bar{u} \Gamma u \bar{d} \Gamma d-\frac{1}{2}\left(\bar{u} \Gamma u \bar{d} \gamma_{5} \Gamma \gamma_{5} d+\bar{d} \Gamma d \bar{u} \gamma_{5} \Gamma \gamma_{5} u-\bar{u}\left\{\gamma_{5}, \Gamma\right\} u \bar{d}\left\{\gamma_{5}, \Gamma\right\} d\right)
$$

Of course, the isotope invariance provides the relations $B^{+}\left((\bar{d} d)^{2}\right)=B^{-}\left((\bar{u} u)^{2}\right)$, etc.

For the important special case $\Gamma=I$ Eqs. (24)-(28) take the form

$$
\begin{aligned}
& B^{ \pm}\left((\bar{u} u)^{2}\right)=-(\bar{u} u)^{2}-\bar{u} u \bar{d} d-\bar{d} \gamma_{5} u \bar{u} \gamma_{5} d \\
& B^{0}\left((\bar{u} u)^{2}\right)=-2\left((\bar{u} u)^{2}+\left(\bar{u} \gamma_{5} u\right)^{2}\right)
\end{aligned}
$$

and

$$
B^{ \pm}(\bar{u} u \bar{d} d)=-\frac{1}{2}\left((\bar{u} u+\bar{d} d)^{2}+2 \bar{u} \gamma_{5} d \bar{d} \gamma_{5} u\right)
$$

while

$$
B^{0}(\bar{u} u \bar{d} d)=-2\left(\bar{u} u \bar{d} d-\bar{u} \gamma_{5} u \bar{d} \gamma_{5} d\right) .
$$

Note that Eqs. (24)-(32) are obtained for the operators $S_{X X}$ defined by Eq.(15), i.e. for the colourless diquarks. The operators $B\left(R_{X X}\right)$ with $R_{X X}$ defined by Eq.(16) are expressed by the same Eqs. (25)-(28) with the matrices $\Gamma$ being replaced by $\widetilde{\Gamma}^{\rho}=\Gamma \lambda^{\rho}$ with further summation over $\rho$.

By using Eqs. (9) and (18) one can obtain the operator $B(A)$ for the four-quark operator of the general form

$$
A=\sum_{\rho} \bar{\Psi} \widetilde{\Gamma}_{i}^{\rho} P_{1} \Psi \cdot \bar{\Psi} \widetilde{\Gamma}_{j}^{\rho} P_{2} \Psi
$$


with $\Psi=\left(\begin{array}{l}u \\ d\end{array}\right)$ being the quark spinor. The operators $P_{1,2}$ are the projection operators in the isospin space. Each of them is equal either to $P_{+}$or to $P_{-}$with $P_{ \pm}=\left(1 \pm \tau_{3}\right) / 2$. We defined

$$
\widetilde{\Gamma}_{k}^{\rho}=\Gamma_{k} \lambda^{\rho}
$$

for any $4 \times 4$ matrix $\Gamma_{k}$ acting on Lorentz indices.

The matrix $\lambda^{\rho}(\rho=0 \ldots 8)$ is either the unit matrix $(\rho=0)$ or one of the Gell-Mann colour matrices $(\rho=1, \ldots 8)$. For the operator $A$ defined by Eq.(33) we obtain

$$
\begin{gathered}
B^{\alpha}=-\frac{1}{2} \bar{\Psi}\left(\gamma_{5} \Gamma_{j}^{\rho} \tau_{\bar{\alpha}} P_{2}+\Gamma_{j}^{\rho} \gamma_{5} P_{2} \tau_{\bar{\alpha}}\right) \Psi \\
\times \bar{\Psi}\left(\gamma_{5} \Gamma_{i}^{\rho} \tau_{\alpha} P_{1}+\Gamma_{i}^{\rho} \gamma_{5} P_{1} \tau_{\alpha}\right) \Psi-\frac{1}{2} \bar{\Psi} \Gamma_{j}^{\rho} P_{2} \Psi \\
\times\left(\bar{\Psi}_{1} \Gamma_{i}^{\rho} \tau_{\bar{\alpha}} \tau_{\alpha} P_{1} \Psi+\bar{\Psi} \Gamma_{i}^{\rho} P_{1} \tau_{\alpha} \tau_{\bar{\alpha}} \Psi\right)+(1 \rightarrow 2)
\end{gathered}
$$

with $\alpha=+,-, 0$ standing for the pion isospin indices. For the specific cases Eq.(35) takes a much more simple form since some of the terms vanish: $\tau_{+} P_{+} \Psi=\tau_{-} P_{-} \Psi=0 ; P_{-} \tau_{+} \Psi=P_{+} \tau_{-} \Psi=0$.

\section{FOUR-QUARK OPERATORS CONTAINING HEAVIER QUARKS}

Here we calculate the expectation values of the operators containing heavier quarks. Calculations, similar to those described in the previous Section, provide

$$
B^{ \pm}\left(\bar{u} \Gamma^{X} u \bar{\psi}_{i} \Gamma^{Y} \psi_{i}\right)=-\frac{1}{2}\left(\bar{u} \Gamma^{X} u+\bar{d} \gamma_{5} \Gamma^{X} \gamma_{5} d\right) \bar{\psi}_{i} \Gamma^{Y} \psi_{i}
$$

while

$$
B^{0}\left(\bar{q} \Gamma^{X} q \bar{\psi}_{i} \Gamma^{Y} \psi_{i}\right)=-\frac{1}{2}\left(\bar{q} \Gamma^{X} q+\bar{q} \gamma_{5} \Gamma^{X} \gamma_{5} q\right) \bar{\psi}_{i} \Gamma^{Y} \psi_{i}
$$

For the operator $A$ consisting of the heavy quarks only, e.g. $A=\bar{\psi}_{i} \Gamma^{X} \psi_{i} \bar{\psi}_{j} \Gamma^{Y} \psi_{j}$, we find immediately

$$
B^{\alpha}(A)=0 .
$$

\section{FACTORIZATION APPROXIMATION}

Now we calculate the rhs of Eqs. (24)-(37) in the factorization approximation [13]. This means that the sum over the intermediate states is assumed to be dominated by the vacuum state, i.e.

$$
\begin{gathered}
\left\langle 0\left|\bar{q}_{\alpha, a}^{i} q_{\beta, b}^{j} \bar{q}_{\gamma, c}^{k} q_{\delta, d}^{\ell}\right| 0\right\rangle=\frac{1}{12^{2}}\left(\delta_{i j} \delta_{\alpha \beta} \delta_{a b} \delta_{k \ell} \delta_{\gamma \delta} \delta_{c d}-\right. \\
\left.-\delta_{i \ell} \delta_{\alpha \delta} \delta_{a d} \delta_{j k} \delta_{\beta \gamma} \delta_{b c}\right)\left\langle 0\left|\bar{q}^{i} q^{i}\right| 0\right\rangle\left\langle 0\left|\bar{q}^{j} q^{j}\right| 0\right\rangle
\end{gathered}
$$

with the upper indices standing for the flavour. For the quarks of the same flavour this provides for any $4 \times 4$ matrices $\Gamma_{r}, \Gamma_{s}$

$$
\left\langle 0\left|\bar{q} \widetilde{\Gamma}_{r}^{\rho} q \bar{q} \widetilde{\Gamma}_{s}^{\rho} q\right| 0\right\rangle=\frac{1}{12^{2}}\left(\operatorname{Sp} \widetilde{\Gamma}_{r}^{\rho} \operatorname{Sp} \widetilde{\Gamma}_{s}^{\rho}-\operatorname{Sp} \widetilde{\Gamma}_{r}^{\rho} \widetilde{\Gamma}_{s}^{\rho}\right)(\langle 0|\bar{q} q| 0\rangle)^{2}
$$

with $\widetilde{\Gamma}_{k}^{\rho}$ defined by Eq.(34). For $\rho=0$, when $\lambda^{\rho}$ is the unit matrix and $\widetilde{\Gamma}_{k}^{0}=\Gamma_{k}$, Eq.(40) takes the form

$$
\left.\left\langle 0\left|\bar{q} \Gamma_{r} q \bar{q} \Gamma_{s} q\right| 0\right\rangle=\frac{1}{16}\left(\operatorname{Sp} \Gamma_{r} \operatorname{Sp} \Gamma_{s}-\frac{1}{3} \operatorname{Sp} \Gamma_{r} \Gamma_{s}\right)\langle 0|\bar{q} q| 0\rangle\right)^{2},
$$

while 


$$
\left\langle 0\left|\sum_{\rho=1} \bar{q} \widetilde{\Gamma}_{r}^{\rho} q \bar{q} \widetilde{\Gamma}_{s}^{\rho} q\right| 0\right\rangle=-\frac{1}{9} \operatorname{Sp} \Gamma_{r} \Gamma_{s}(\langle 0|\bar{q} q| 0\rangle)^{2}
$$

For the quarks with different flavours $i \neq j$ we find

$$
\left\langle 0\left|\bar{q}_{i} \Gamma_{r} q_{i} \bar{q}_{j} \Gamma_{s} q_{j}\right| 0\right\rangle=\frac{1}{16} \operatorname{Sp} \Gamma_{r} \operatorname{Sp} \Gamma_{s}\left\langle 0\left|\bar{q}_{i} q_{i}\right| 0\right\rangle\left\langle 0\left|\bar{q}_{j} q_{j}\right| 0\right\rangle
$$

and

$$
\left\langle 0\left|\bar{q}_{i} \Gamma_{r} q_{j} \bar{q}_{j} \Gamma_{s} q_{i}\right| 0\right\rangle=-\frac{1}{3 \cdot 16} \operatorname{Sp} \Gamma_{r} \Gamma_{s}\left\langle 0\left|\bar{q}_{i} q_{i}\right| 0\right\rangle\left\langle 0\left|\bar{q}_{j} q_{j}\right| 0\right\rangle
$$

while

$$
\left\langle 0\left|\sum_{\rho=1} \bar{q}_{i} \widetilde{\Gamma}_{r}^{\rho} q_{j} \bar{q}_{j} \widetilde{\Gamma}_{s}^{\rho} q_{i}\right| 0\right\rangle=-\frac{1}{9} \operatorname{Sp} \Gamma_{r} \Gamma_{s}\left\langle 0\left|\bar{q}_{i} q_{i}\right| 0\right\rangle\left\langle 0\left|\bar{q}_{j} q_{j}\right| 0\right\rangle
$$

which is true for $i=j$ as well, and

$$
\left\langle 0\left|\sum_{\rho=1} \bar{q}_{i} \widetilde{\Gamma}_{r}^{\rho} q_{i} \bar{q}_{j} \widetilde{\Gamma}_{s}^{\rho} q_{j}\right| 0\right\rangle=0
$$

Assuming also isospin invariance, i.e. $\langle 0|\bar{u} u| 0\rangle=\langle 0|\bar{d} d| 0\rangle=\langle 0|\bar{q} q| 0\rangle$ we find

$$
\begin{aligned}
& \left\langle 0\left|B^{+}\left((\bar{u} \Gamma u)^{2}\right)\right| 0\right\rangle=-\frac{1}{8}\left((\operatorname{Sp} \Gamma)^{2}-\frac{1}{3} \mathrm{Sp} \Gamma^{2}\right)(\langle 0|\bar{q} q| 0\rangle)^{2} \\
& \left\langle 0\left|B^{-}\left((\bar{u} \Gamma u)^{2}\right)\right| 0\right\rangle=\left\langle 0\left|B^{+}\left((\bar{u} \Gamma u)^{2}\right)\right| 0\right\rangle
\end{aligned}
$$

while

$$
\left\langle 0\left|B^{0}\left((\bar{u} \Gamma u)^{2}\right)\right| 0\right\rangle=-\frac{1}{8}\left((\operatorname{Sp} \Gamma)^{2}-\frac{1}{3} \operatorname{Sp} \Gamma^{2}-\frac{1}{3} \operatorname{Sp} \Gamma \gamma_{5} \Gamma \gamma_{5}+\left(\operatorname{Sp} \gamma_{5} \Gamma\right)^{2}\right) \cdot(\langle 0|\bar{q} q| 0\rangle)^{2}
$$

For the mixed operator $A=\bar{u} \Gamma u \bar{d} \Gamma d$ we obtain

$$
\left\langle 0\left|B^{ \pm}\right| 0\right\rangle=-\frac{1}{8}\left((\operatorname{Sp} \Gamma)^{2}-\frac{1}{3} \operatorname{Sp} \Gamma \gamma_{5} \Gamma \gamma_{5}\right)(\langle 0|\bar{q} q| 0\rangle)^{2}
$$

and

$$
\left\langle 0\left|B^{0}\right| 0\right\rangle=-\frac{1}{8}\left((\operatorname{Sp} \Gamma)^{2}-\left(\operatorname{Sp} \gamma_{5} \Gamma\right)^{2}\right) \cdot(\langle 0|\bar{q} q| 0\rangle)^{2}
$$

We present also the result for the operators containing the coloured diquarks. For the operator $\sum_{\rho}\left(\bar{u} \Gamma \lambda^{\rho} u\right)^{2}$ they are

$$
\begin{aligned}
\left\langle 0\left|B^{+}\right| 0\right\rangle & =\frac{2}{9} \operatorname{Sp} \Gamma^{2}(\langle 0|\bar{q} q| 0\rangle)^{2} \\
\left\langle 0\left|B^{-}\right| 0\right\rangle & =\frac{2}{9} \operatorname{Sp} \Gamma^{2}(\langle 0|\bar{q} q| 0\rangle)^{2} \\
\left\langle 0\left|B^{0}\right| 0\right\rangle & =\frac{2}{9}\left(\operatorname{Sp} \Gamma^{2}+\operatorname{Sp} \Gamma \gamma_{5} \Gamma \gamma_{5}\right)(\langle 0|\bar{q} q| 0\rangle)^{2},
\end{aligned}
$$

while for $A=\sum_{\rho} \bar{u} \Gamma \lambda^{\rho} u \bar{d} \Gamma \lambda^{\rho} d$

$$
\left\langle 0\left|B^{ \pm}\right| 0\right\rangle=\frac{2}{9} \operatorname{Sp} \Gamma \gamma_{5} \Gamma \gamma_{5}(\langle 0|\bar{q} q| 0\rangle)^{2} ; \quad\left\langle 0\left|B^{0}\right| 0\right\rangle=0 .
$$

Equations (47)-(55) are true for any $4 \times 4$ matrix $\Gamma$. If $\Gamma=\Gamma^{X}$ with $\Gamma^{X}$, being one of the basic matrices defined by Eq.(14), the unit matrix $\Gamma=\Gamma^{1}$ is the only one for which $\mathrm{Sp} \Gamma \neq 0$. Thus the scalar condensate appears to be numerically larger than the other condensates of the form $A=\left(\bar{q} \Gamma^{X} q\right)^{2}$. The values are 


$$
\begin{aligned}
\left\langle 0\left|B^{ \pm}\left((\bar{q} q)^{2}\right)\right| 0\right\rangle & =-\frac{22}{12}(\langle 0|\bar{q} q| 0\rangle)^{2} \\
\left\langle 0\left|B^{0}\left((\bar{q} q)^{2}\right)\right| 0\right\rangle & =-\frac{20}{12}(\langle 0|\bar{q} q| 0\rangle)^{2},
\end{aligned}
$$

while for $A=\bar{u} u \bar{d} d$

$$
\begin{aligned}
& \left\langle 0\left|B^{ \pm}\right| 0\right\rangle=-\frac{22}{12}(\langle 0|\bar{q} q| 0\rangle)^{2} \\
& \left\langle 0\left|B^{0}\right| 0\right\rangle=-2(\langle 0|\bar{q} q| 0\rangle)^{2} .
\end{aligned}
$$

Deviations of the coefficients in the rhs of Eqs (56)-(58) manifest themselves in the units of the characteristic factor $1 / 12$-see Eq (41). Also, for the operator $A=\sum_{\rho}\left(\bar{u} \lambda^{\rho} u\right)^{2}$

$$
\left\langle 0\left|B^{ \pm}\right| 0\right\rangle=\frac{8}{9}(\langle 0|\bar{q} q| 0\rangle)^{2} ; \quad\left\langle 0\left|B^{0}\right| 0\right\rangle=\frac{16}{9}(\langle 0|\bar{q} q| 0\rangle)^{2},
$$

while for $A=\sum_{\rho} \bar{u} \lambda^{\rho} u \bar{d} \lambda^{\rho} d$

$$
\left\langle 0\left|B^{ \pm}\right| 0\right\rangle=\frac{8}{9}(\langle 0|\bar{q} q| 0\rangle)^{2} ; \quad\left\langle 0\left|B^{0}\right| 0\right\rangle=0 .
$$

For the expectation values of the operators containing heavier quarks we obtain for the operator $A=\bar{q} \Gamma^{X} q \bar{\psi}_{i} \Gamma^{Y} \psi_{i}$

$$
\langle 0|B| 0\rangle=-\frac{1}{32}\left(\operatorname{Sp} \Gamma^{X} \operatorname{Sp} \Gamma^{Y}+\operatorname{Sp} \Gamma^{X} \operatorname{Sp} \gamma_{5} \Gamma^{Y} \gamma_{5}\right)\langle 0|\bar{q} q| 0\rangle\left\langle 0\left|\bar{\psi}_{i} \psi_{i}\right| 0\right\rangle,
$$

while $\langle 0|B| 0\rangle=0$ for the operators containing the coloured diquarks.

\section{SUMMARY}

We solved the problem of expressing the expectation values of four-quark operators in pions through those in vacuum. The further specific calculations were carried out in the framework of the factorization hypothesis.

For the scalar operators of the light quarks the expectation values turn out to be negative. This can be understood in the following way. Note that the four-quark condensates take the form of the superposition of the products of the two-quark operators $\eta_{1,2}$, i.e., $A=\eta_{1} \eta_{2}$. For $\eta_{1}=\eta_{2}=\eta$ Eq.(5) can be presented as

$$
\left\langle h\left|\eta^{2}\right| h\right\rangle=2\langle 0|\eta| 0\rangle\langle h|\eta| h\rangle+\left\langle h\left|\int d^{3} x(\eta(x)-\langle 0|\eta| 0\rangle)^{2}\right| h\right\rangle,
$$

if the factorization approximation is assumed.

The first term in the rhs of Eq.(63) describes the "disconnected contribution" with one of $\bar{q} q$ pairs coming from vacuum. For the expectation values of the operators $(\bar{q} q)^{2}$, i.e. $\eta=\bar{q} q$, the first term in the rhs of Eq.(63) is exactly the rhs of Eq.(4). Thus the expectation values of operators $(\bar{q} q)^{2}$ are dominated by "disconnected terms". The same refers to the operator $A=\bar{u} u \bar{d} d$. For the operators $\eta$ of the form $\bar{q} \Gamma^{X} q$ with $X \neq 1$ (see Eq.(14)) the "disconnected terms " vanish since the scalar condensate is the only one with a nonzero vacuum expectation value.

For the scalar operators the second term of rhs of Eq.(63) describes the correlation between $\bar{q} q$ pairs inside the pion. It corresponds to deviations of the rhs of Eqs. (56)-(59) from that of Eq.(4). If these pairs would have been independent, we would expect the estimation

$$
\langle 0|B| 0\rangle-(-2) \cdot \frac{\langle 0|\bar{q} q| 0\rangle^{2}}{f_{\pi}^{2}} \approx\langle\pi|\bar{q} q| \pi\rangle \cdot \frac{n_{\pi}^{q}}{V_{\pi}}
$$

to be true. Here $n_{\pi}^{q}$ being the number of $\bar{q} q$ pairs of the certain flavour (see Eq.(12)), while $V_{\pi}$ is the pion volume. The latter can be obtained since the pion radius is known to be 20]

$$
r_{\pi}=\frac{\sqrt{3}}{2 \pi f_{\pi}} .
$$


However, the value obtained from Eqs. (56)-(59) appears to be much smaller than the rhs of Eq(64). This means that the pairs of light quarks are correlated strongly and that the probability of finding two pairs at the same point is much smaller than in the approximation of independent pairs.

As one can see from the formulae of Sec.5, for the condensates of the same flavour $\bar{q} \Gamma^{X} q \bar{q} \Gamma^{X} q$ those with $X=1$ are about 10 times larger than the other values of $X$. In other words, the value of the scalar condensate is about 10 times larger than the values of the vector, pseudoscalar, pseudovector and tensor condensates. This is because $\Gamma^{1}$ is the only matrix with $\operatorname{Sp} \Gamma^{X} \neq 0$. Since for each of the matrices $\Gamma^{X}$ defined by Eq.(14) $\left|\operatorname{Sp}\left(\Gamma^{\mathrm{X}}\right)^{2}\right|=4$, the condensates with $X \neq 1$ contain the numerically small factor $\frac{1}{3 \cdot 4}=\frac{1}{12}$. For the condensates with different flavours $\bar{u} \Gamma^{X} u \bar{d} \Gamma^{X} d$ the scalar condensate dominates for the charged pions. For the neutral pions the pseudoscalar condensate is as large as the scalar condensate.

We obtain also the values for the condensates containing heavier quarks. Assuming $\langle 0|\bar{s} s| 0\rangle \approx\langle 0|\bar{q} q| 0\rangle$ with $q$ standing for the " $u$ " or " $d "$ quark, we find the expectation values $\langle\pi|\bar{q} q \bar{s} s| \pi\rangle$ to be only about twice smaller than the value $\left\langle\pi\left|(\bar{q} q)^{2}\right| \pi\right\rangle$. The expectation values, containing two pairs of heavier quarks (s, c, etc.) become zero. The expectation values of the four-quark operators in pions in the terms describing the four-quark interactions in NJL model are zero. This is the expected result corresponding to the degeneracy of the ground state before the spontaneous symmetry breaking.

We thank M. Eides and T. Gutsche for useful discussions and E.Gerjuoy for reading the manuscript. Two of us (E. G. D.) and (V. A. S.) are grateful for the hospitality of University of Tuebingen during their visits. The work was supported by DFG grant No.438 RUS 113/595/0-1 and by RFBR grant No.00-02-16853. 
[1] S. Weinberg, in: "A Festschrift for I.I. Rabi", L. Motz, ed., N.Y. Academy of Science, N.Y., 1977.

[2] M. Anselmino and S. Forte, Z. Phys. C61 (1994) 453.

[3] S. Forte, Phys. Rev. D47 (1993) 1842.

[4] J. Gasser, Ann. Phys. 136 (1981) 62.

[5] E.G. Drukarev and E.M. Levin, Prog.Part.Nucl.Phys. 27 (1991) 77.

[6] T.D. Cohen, R.J. Furnstahl, D.K. Griegel, and X. Jin, Prog.Part.Nucl.Phys. 35 (1995) 221.

[7] L.S. Celenza, C.M. Shakin, Wei-Dong Sun and J. Szweda, Phys. Rev. C51 (1995) 937.

[8] M.B. Johnson and L.S. Kisslinger, Phys.Rev. C52 (1995) 1022.

[9] A.W. Thomas, Adv. Nucl. Phys. 13 (1984) 1;

[10] Th . Gutsche and D. Robson, Phys. Lett. B229 (1989) 333;

V. E. Lyubovitskij, Th. Gutsche and Amand Faessler, hep-ph/0105044 (Invited talk at Workshop on the Physics of Exited Nucleons (NSTAR 2001), Mainz, Germany, 7-10 March 2001).

[11] H. Lehmann, K. Symanzik and W. Zimmerman, Nuovo Com. 6 (1957) 319.

[12] F.J. Yundurain, "Quantum Chromodynamics", Springer-Verlag, 1983.

[13] M.A. Shifman, A.I. Vainshtein, and V.I. Zakharov, Nucl.Phys. B147 (1979) 385.

[14] M.A. Shifman, "ITEP Lectures on particle physics and field theory", v.1, Singapore, World Scientific, 1999.

[15] M. Gell-Mann, R.J. Oakes, and B. Renner, Phys.Rev. 175 (1968) 2195.

[16] Y. Nambu and G. Jona-Lasinio, Phys. Rev. 122 (1961) 345.

[17] G.t'Hooft, Phys.Rev.D 14 (1976) 3432.

[18] U. Vogl and W. Weise, Prog.Part.Nucl.Phys. 27 (1991) 195;

K. Tsushima, T.Maruyama, and A.Faessler, Nucl.Phys. A 535 (1991) 497;

T.Maruyama, K. Tsushima, and A.Faessler, Nucl.Phys. A 537 (1992) 303.

[19] T. Hatsuda, T. Kunihiro, Phys. Rep. 247 (1994) 221.

[20] R.D. Carlitz and D.B. Creamer, Ann. Phys. 118 (1979) 429. 